\title{
Validation of a HPLC method for flavonoid biomarkers in skullcap (Scutellaria) and its use to illustrate wide variability in the quality of commercial tinctures.
}

\author{
Jiayu Gao, Alberto Sanchez-Medina, Barbara A. Pendry, Michael J. Hughes, Geoffrey P. Webb and \\ Olivia Corcoran
}

The Medicines Research Group, School of Health and Bioscience, University of East London, Stratford, London UK.

Received, January 16, 2008; Revised, March 14, 2008; Accepted, March 17, 2008; Published March 19, 2008.

\begin{abstract}
PURPOSE. To compare the flavonoid biomarker content (baicalin, baicalein and wogonin) of eleven commercial tinctures derived from Scutellaria lateriflora aerial parts $(\mathrm{n}=7)$ and Scutellaria baicalensis root $(\mathrm{n}=4) . S$. lateriflora tinctures are used by Western herbal practitioners to treat anxiety whereas $S$. baicalensis tinctures are used to treat inflammatory disease. METHODS. Baicalin and baicalein were purchased from Aldrich Chemical Co. and Wogonin was purchased from ChromaDex. The internal standard (4-hydroxybenzoic acid) was obtained from Acros Organics. The column used was a Luna $\mathrm{C}_{18}, 5 \mu \mathrm{m}$ $(150 \times 4.6 \mathrm{~mm}$, Phenomenex) maintained at ambient room temperature. A HP1050 HPLC system was used, comprising a gradient pump with degasser, a variable wavelength UV detector set to $270 \mathrm{~nm}$, and an autosampler. Gradient elution was performed using $0.1 \%$ formic acid (eluent A) and methanol (eluent B). The gradient elution initial conditions were $45 \%$ B with linear gradient to $60 \%$ from 2 to $10 \mathrm{~min}$, followed by linear gradient to $70 \% \mathrm{~B}$ at $30 \mathrm{~min}$, and then linear gradient to $99 \% \quad \mathrm{~B}$ at $31 \mathrm{~min}$, this proportion being maintained for $1 \mathrm{~min}$. The mobile phase was then returned to initial conditions at $33 \mathrm{~min}$ and maintained until the end of the run at $35 \mathrm{~min}$. The flow rate was $1 \mathrm{~mL} / \mathrm{min}$. The assay was validated for sensitivity, accuracy and reproducibility. RESULTS. The concentration range of biomarkers (baicalin, baicalein and wogonin) in commercial tinctures is reported for S. lateriflora (baicalin: 0-12.66 $\mathrm{mg} / \mathrm{mL}$; baicalein: $0-0.63 \mathrm{mg} / \mathrm{mL}$; wogonin: $0-0.16 \mathrm{mg} / \mathrm{mL}$ ) and for S. baicalensis (baicalin: $0.12-10.61 \mathrm{mg} / \mathrm{mL}$; baicalein: $0.52-5.88 \mathrm{mg} / \mathrm{mL}$; wogonin: $0.08-1.61 \mathrm{mg} / \mathrm{mL}$ ). CONCLUSION. The wide variability in biomarker concentrations between commercial tinctures has important implications for the manufacturers of commercial tinctures, for herbal practitioners in the choice of
\end{abstract}

tinctures and not least for pharmacology and clinical researchers.

\section{INTRODUCTION}

The genus Scutellaria consists of over 350 species worldwide and has been used by many cultures to treat a variety of medical conditions, including anxiety, nervous disorders, liver disease and cancers (1). Two species of particular interest are S. lateriflora Labiatae (American skullcap) and $S$. baicalensis Georgi (Chinese skullcap). The aerial parts of $S$. lateriflora, a native of North America, have a long tradition of use in Western herbal medicine as a relaxing nervine for sleep disorders and nervous conditions where stress and worry cause muscular tension. S. baicalensis is grown in China and Russia where root decoction has traditionally been used in Chinese medicine as a cancer treatment, remedy for inflammation, allergic disorders, hyperlipidemia and atherosclerosis (1). Western herbal practitioners use tinctures (hydroalcoholic solutions) as a common formulation due to ease of storage and dosage (2). These practitioners use tinctures of $S$. lateriflora aerial parts in the treatment of anxiety symptoms (including insomnia, anorexia nervosa and tension headache) and muscle spasms (including fibromyalgia, seizure disorders and mild Tourette's syndrome) (1). A common daily dose of liquid extract ( $1: 1$ in $25 \%$ ethanol) is 15-30 drops, equivalent to $2.0-4.0 \mathrm{~mL}$ per day and the daily dose of tincture (1:5 in $45 \%$ alcohol) is 1-2 mL (3).

Corresponding Author: Dr. Olivia Corcoran, The Medicines Research Group, School of Health and Bioscience, University of East London, Romford Road, Stratford, London, E-mail o.corcoran@uel.ac.uk 
Although the flavonoids baicalin and baicalein (Figure 1) have previously been identified in $S$. lateriflora, anxiolytic activity of S. lateriflora was reported only for aqueous extracts, containing predominantly GABA amino acids, which were not quantified in this study (4).

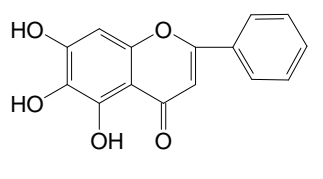

1
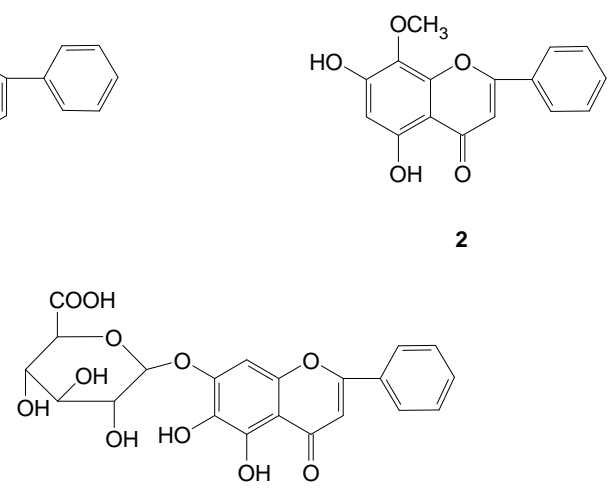

3

Figure 1. Flavonoid biomarkers in Scutellaria: 1) Baicalein, 2) Wogonin and 3) Baicalin.

The potential efficacy of standardised extracts of $S$. lateriflora as an anxiolytic in humans is claimed by a double blind, placebo-controlled clinical study of healthy adults $(\mathrm{n}=15$ females and $\mathrm{n}=4$ males, aged from 20-70 yrs) stating that skullcap reduced symptoms of anxiety and tension in a dose-dependent manner for $120 \mathrm{~min}$ after acute administration of $200 \mathrm{mg}$ freeze-dried extract compared with control (5). Otherwise, there is scant data on constituents of S. lateriflora extracts and scant scientific evidence of anxiolytic activity.

By comparison to S. lateriflora, the chemical constituents of $S$. baicalensis root are welldocumented. S. baicalensis is one of the most widely used traditional herbal medicines in both Eastern and Western medicine by decoction (boiling plant material) or tinctures, respectively. Chinese medical herbalists use decoctions primarily in cancer treatment whereas Western medical herbalists use tinctures in anti-inflammatory, antibacterial, and anti-allergy therapy. A typical daily dose of $S$. baicalensis tincture is $15-30$ drops, equivalent to $2.0-4.5 \mathrm{~mL}$ per day of $1: 5$ in $45 \%$ alcohol (3). Three important biomarkers isolated to date are the flavonoids, baicalin, baicalein, and wogonin (1). Baicalin has been reported as having various pharmacological properties including anti-tumor (6-8) anti-allergic (9-11), anti-inflammatory $(12,13)$, antioxidant and radical scavenging activities (14-16). Baicalein presents the anti-HIV (17), anti-tumor (8), antioxidant and radical scavenging abilities (14-16). Wogonin possesses anti-tumor $(8,18)$, anti-inflammatory (12), anti-hepatitis B virus (19), antioxidant and radical scavenging abilities (14).

However, a concern for Western herbal practitioners is that commercial tinctures of both Scutellaria species vary widely in the drug-to-extract ratio (ranging from 1:1 to 1:5) and alcohol concentration ( 25 to $70 \%$ ), depending on the manufacturer. Though S. lateriflora and $S$. baicalensis have demonstrable value as herbal therapy on the basis of continued traditional success, one unanswered question is the optimal choice of tincture composition with respect to drug-to-extract ratio and alcohol concentration. Whereas the quantitative and qualitative determination of a pharmaceutical component is critical in all stages of drug production, unfortunately in the herbal industry there is still no agreed uniform criterion for the production of tinctures. Previous work in our laboratory has shown wide variability in biomarker content (rosmarinic acid) for lemon balm (Melissa officinalis) (20). Thus, for the herbalist there is no guarantee or measure of either quality or efficacy from the products currently available. The main aims of this study are threefold. Firstly to develop and validate methods for the quantification of the three key flavonoid biomarkers (baicalin, baicalein, and wogonin) in commercial Scutellaria tinctures. Secondly to enable preliminary judgement to be made on the methods of commercial preparation of the herb likely to maximise the biomarker content of the end product. Thirdly to demonstrate to herbal practitioners, researchers, reviewers and manufacturers the extent of the variation in the biomarker content of commercially available herbal medicines and dietary supplements. This variation could help to explain the variable efficacy of herbal medicines used in clinical practice and also the variation in the reported activity of herbal medicines and dietary supplements in clinical trials and in assays of pharmacological activity $(21,22)$. 


\section{MATERIALS AND METHODS}

\section{Chemicals}

Standards of baicalin and baicalein were purchased from Aldrich Chemical Co. (Dorset, UK). Wogonin was purchased from ChromaDex, Irvine, California. The internal standard (4-hydroxybenzoic acid) was purchased from Acros Organics. (Leicestershire, UK). Formic Acid (Laboratory reagent grade) and methanol (HPLC grade) were purchased from Fisher Scientific Limited (Leicestershire, UK). Water was purified using a Purelab Option System from ELGA (Buckinghamshire, UK). 0.1\% formic acid solution was prepared.

\section{Tinctures}

Sample tinctures of $S$. lateriflora and $S$. baicalensis were purchased anonymously from five companies, representing the range of products commonly used by herbal practitioners in the UK. These Test formulations from a particular manufacturer bore the same lot number and had an expiration date no earlier than 2 years from the date of purchase. The samples were randomly labeled SL1-7 (S. lateriflora) and SB1-4 (S. baicalensis), so their identity was not known until after the analysis was complete.

\section{Sample preparation for HPLC}

Eleven commercial tinctures (Samples SL1-7 and SB1-4) of Scutellaria (S. lateriflora and $S$. baicalensis) were used in this study. Comparison of baicalin, baicalein, and wogonin content was standardised on the basis of milligrams of baicalin, baicalein and wogonin per $\mathrm{mL}$ of tincture. $50 \mu \mathrm{L}$ of tincture was added to $950 \mu \mathrm{L}$ of methanol. 100 $\mu \mathrm{L}$ of each solution were added to $100 \mu \mathrm{L}$ of 4-hydroxybenzoic acid $(1000 \mu \mathrm{g} / \mathrm{mL}$ in methanol) solution. The resultant solutions were centrifuged at $13,000 \mathrm{~min}^{-1}$ for $5 \mathrm{~min}$ before injecting $10 \mu \mathrm{L}$ on the HPLC system. Samples were freshly prepared daily.

\section{HPLC instrumentation}

The quantification of samples and the calibration curves were performed using a HP1050 series HPLC system comprising a gradient pump with degasser, a variable wavelength UV detector and an autosampler. The UV detector was set at 270 $\mathrm{nm}$ and data was acquired using Totalchrom software (Massachusetts, USA) via a Perkin Elmer NCI902 data collector. The column used was a Luna $\mathrm{C}_{18}, 5 \mu \mathrm{m}(150 \mathrm{x} \quad 4.6 \mathrm{~mm}$, Phenomenex) maintained at ambient room temperature. Gradient elution of the samples and standard were performed using $0.1 \%$ formic acid (eluent A) and methanol (eluent B). The gradient elution initial conditions were $45 \%$ of eluent B with linear gradient to $60 \%$ from 2 to $10 \mathrm{~min}$, followed by linear gradient to $70 \%$ of eluent B at $30 \mathrm{~min}$, and then linear gradient to $99 \%$ of eluent $\mathrm{B}$ at $31 \mathrm{~min}$, this proportion being maintained for $1 \mathrm{~min}$. The column was then returned to the initial condition at $33 \mathrm{~min}$ and maintained until the end of the run at $35 \mathrm{~min}$. The flow rate was $1 \mathrm{~mL} / \mathrm{min}$. The sample injection volume was $10 \mu \mathrm{L}$ : three injections were performed for each sample and standard.

\section{Quantitation of baicalin, baicalein and wogonin}

Baicalin, baicalein and wogonin calibration curves $(\mathrm{n}=6)$ were obtained by preparing stock solutions $(1000 \mu \mathrm{g} / \mathrm{mL})$ in methanol. Subsequent dilutions were made to give $500,400,300,200,100$ and 50 $\mu \mathrm{g} / \mathrm{mL} .100 \mu \mathrm{L}$ of each solution were added to 100 $\mu \mathrm{L}$ of 4-hydroxybenzoic acid, IS $(1000 \mu \mathrm{g} / \mathrm{mL}$ in methanol) solution. After mixing, $10 \mu \mathrm{L}$ of each final concentration were injected in triplicate. Stock solutions and dilutions were prepared fresh daily. The linearity of the calibration curves was determined $\left(\mathrm{r}^{2}>0.998\right)$. The limit of detection (LOD) was calculated based on a signal-to-noise ratio $(\mathrm{S} / \mathrm{N}$ : 3.3$)$. The limit of quantitation (LOQ) was then calculated based on the signal-to-noise ratio $(\mathrm{S} / \mathrm{N}$ : 10$)$ using the lowest concentration in the calibration and the highest noise observed when injecting a blank (23). The intraday precision was calculated by comparing the ratio of the area of standard/area of internal standard obtained for 3 injections of standards within the day. For the interday precision, the ratios of the area of standard/area of internal standard in the calibration curve for three days were summed and compared. All biomarker concentrations are quoted in $\mu \mathrm{g} / \mathrm{mL}$, mean \pm standard deviation $(\mathrm{SD}), \mathrm{n}=3$.

\section{RESULTS}

\section{HPLC-UV determination of baicalin, baicalein and wogonin}

The HPLC-UV ${ }_{270 \mathrm{~nm}}$ chromatograms for samples SB1-4 and SL1-7 are illustrated in Figures 2 and 3. 


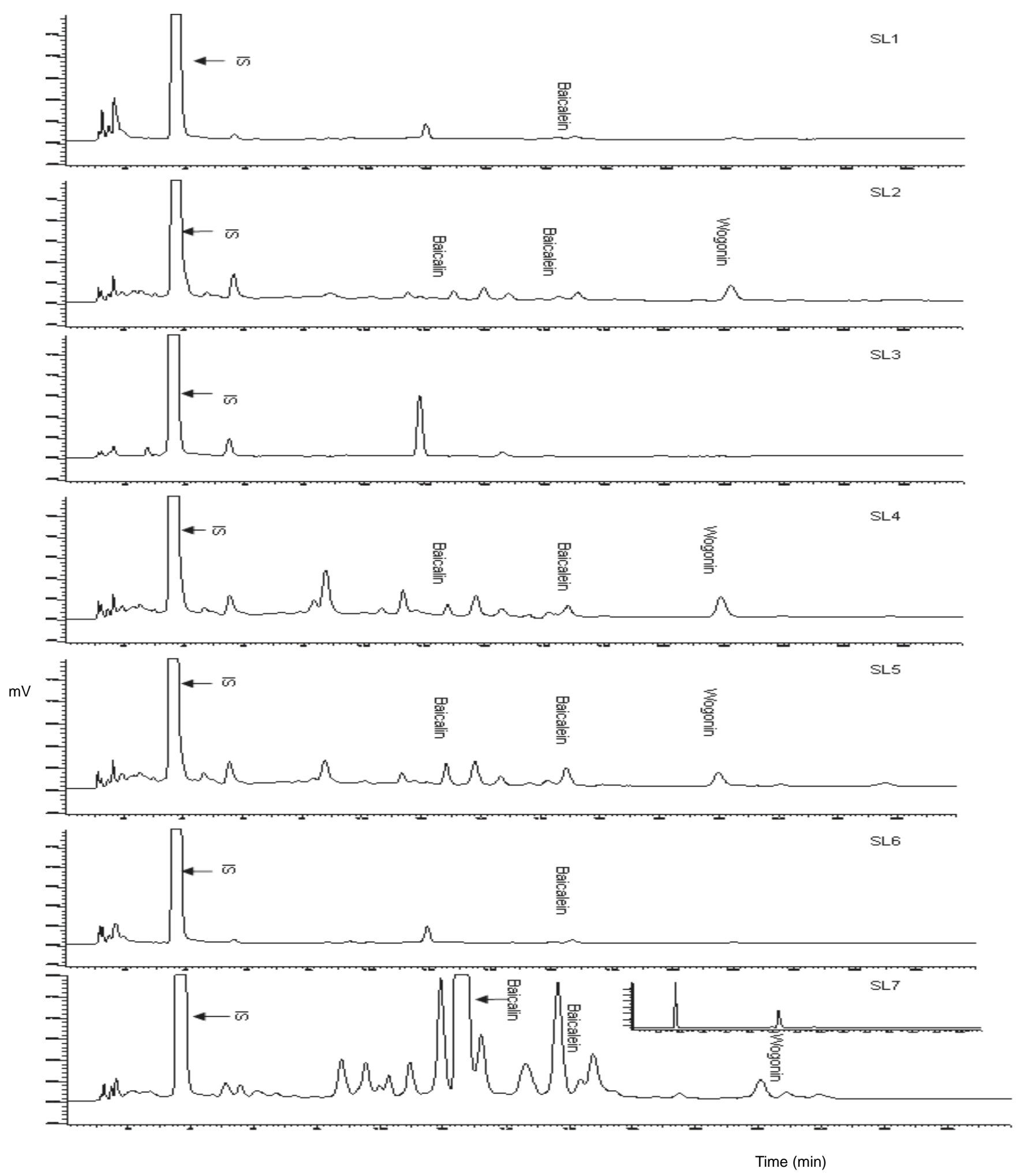

Figure 2 HPLC-UV $270 \mathrm{~nm}$ chromatograms of commercial tinctures produced from S. lateriflora (SL1-7). Retention times of 4-hydroxybenzoic acid (IS), baicalin, baicalein and wogonin were observed at 3.6, 12.6, 16.6 and 21.9 min respectively 


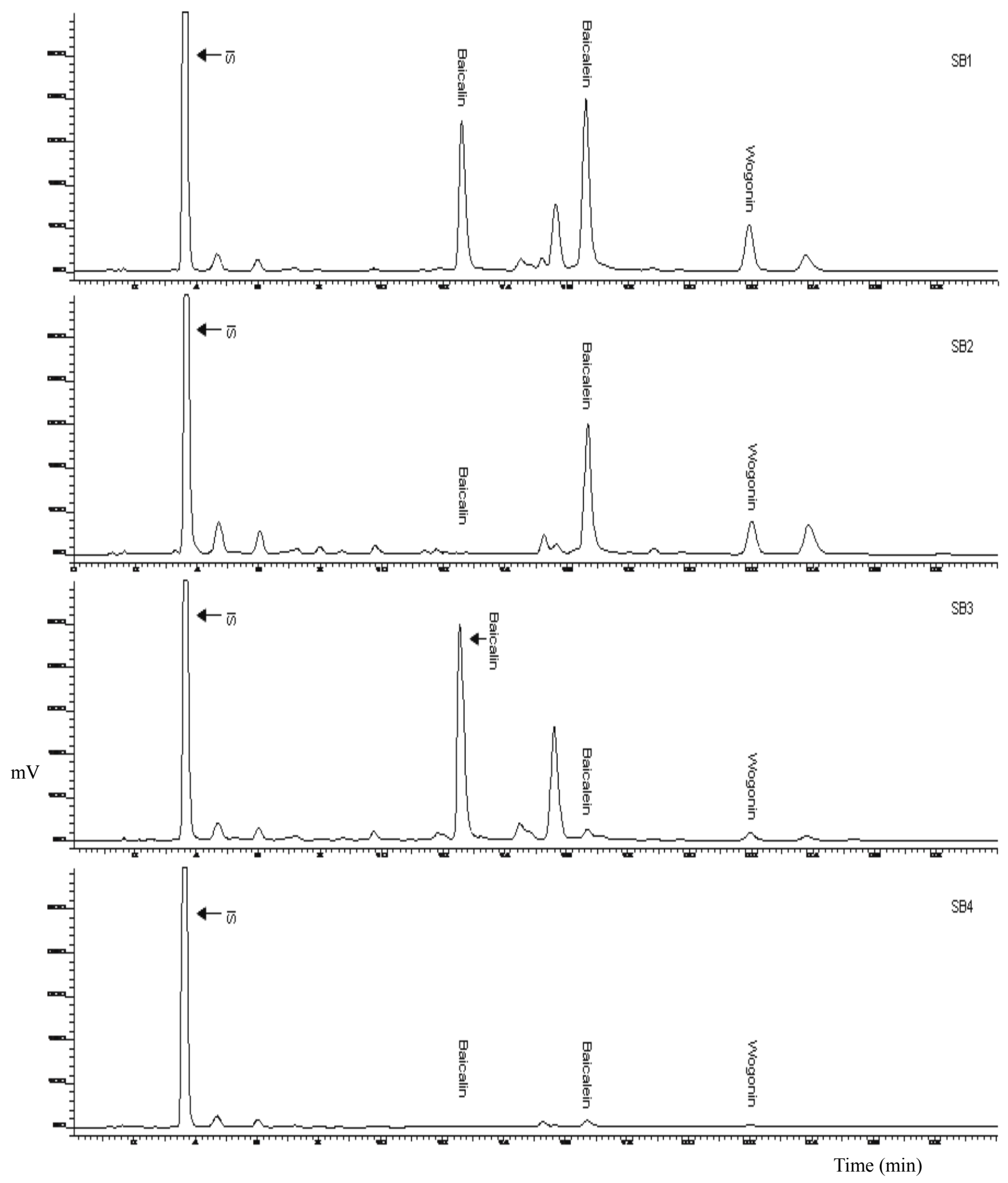

Figure 3 HPLC-UV $270 \mathrm{~nm}$ chromatograms of commercial tinctures produced from S. baicalensis (SB1-4). Retention times of 4-hydroxybenzoic acid (IS), baicalin, baicalein and wogonin were observed at 3.6, 12.6, 16.6 and 21.9 min respectively. 
Retention times $\left(\mathrm{t}_{\mathrm{R}}\right)$ were 3.6, 12.6, 16.6 and 21.9 $\min$ for 4-hydroxybenzoic acid, baicalin, baicalein and wogonin, respectively. The quantitation of baicalin, baicalein and wogonin was achieved using peak area ratios of baicalin, baicalein and wogonin to internal standard. The internal standard 4-hydroxybenzoic acid was chosen because previous injections of all the samples showed no major peaks at the observed $t_{R}$ of 4-hydroxybenzoic acid $(3.6 \mathrm{~min})$. The histograms in Figures 4 and 5 illustrate the resulting concentrations of baicalin, baicalein and wogonin among the 11 commercial preparations.
The amount $(\mu \mathrm{g} / \mathrm{mL}$ Mean $\pm \mathrm{SD})$ of baicalin, baicalein and wogonin in the commercial tinctures is given in Table 1.

Linearity of the calibration curves was determined $\left(\mathrm{r}^{2}>0.998\right)$ confirming the linearity of the analytical method. The intraday precision was calculated by comparing the ratio of the area of baicalin, baicalein and wogonin /area of internal standard (in triplicate) at the mid-point of the calibration curve $(0.2 \mathrm{mg} / \mathrm{mL})$ within the day and the resulting coefficient of variation obtained $(\% \mathrm{CV})$ was $0.03 \%, \quad 0.17 \%$ and $0.10 \%$ respectively.

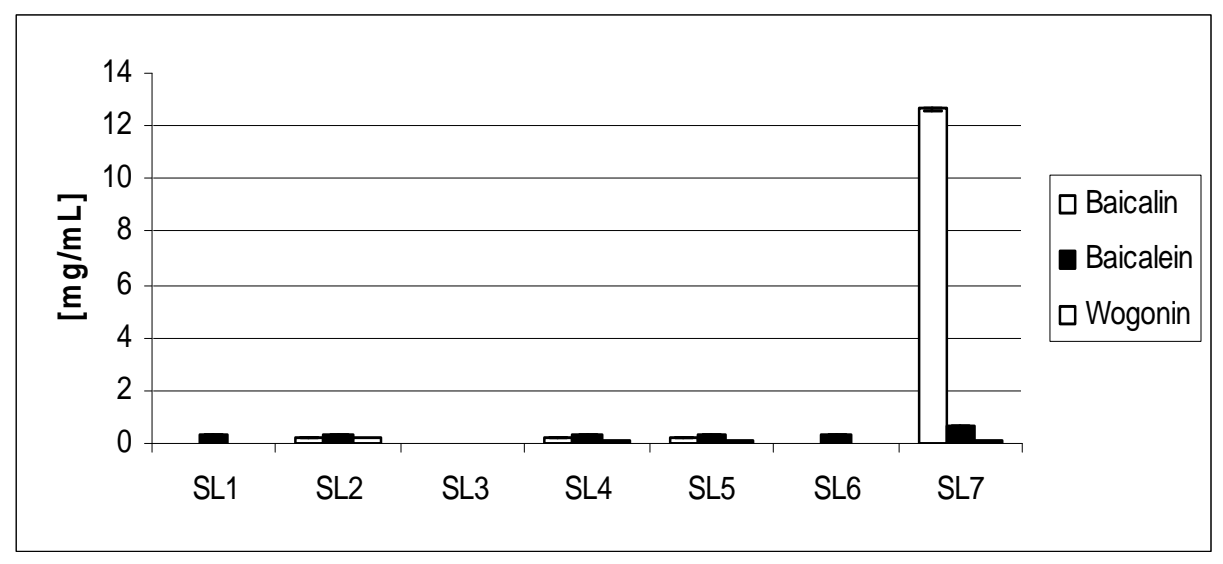

Figure 4 Baicalin, baicalein and wogonin concentrations $(\mu \mathrm{g} / \mathrm{mL}$, mean $\pm \mathrm{SD}, \mathrm{n}=3$ measurements) in commercial $S$. lateriflora tinctures (SL1-7).

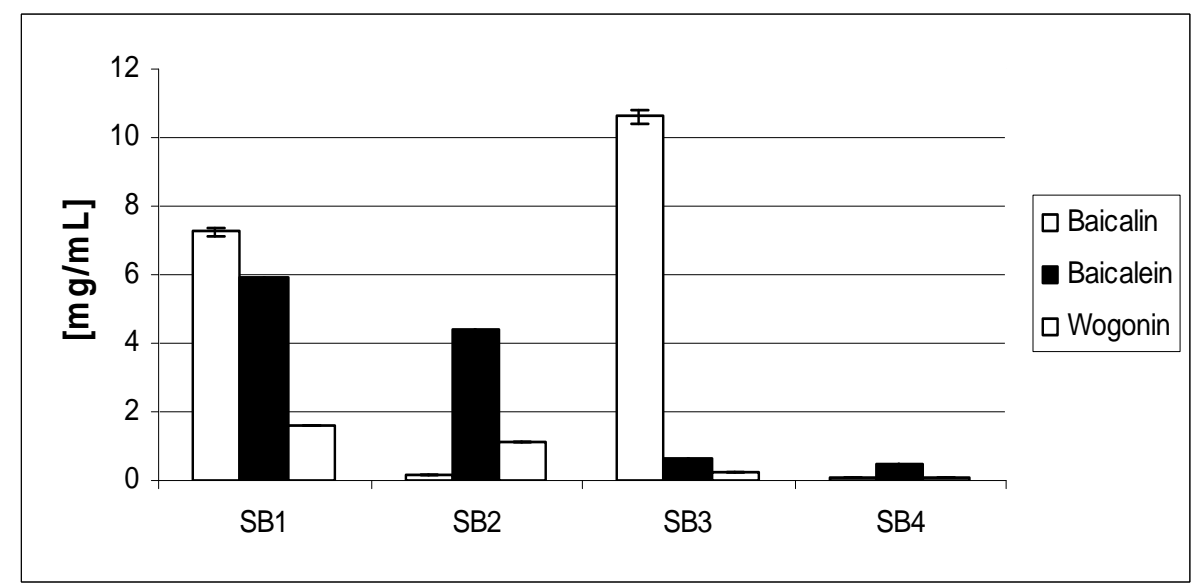

Figure 5 Baicalin, baicalein and wogonin concentrations ( $\mu \mathrm{g} / \mathrm{mL}$, mean $\pm \mathrm{SD}, \mathrm{n}=3$ measurements) in commercial $S$. baicalensis tinctures (SB1-4). 
For the interday precision, the ratio of the area of baicalin, baicalein and wogonin/area of IS for the mid-point in the calibration curve $(0.2 \mathrm{mg} / \mathrm{ml})$ for three different days were added up and compared. The resulting \% CV obtained was $0.36 \%, 0.62 \%$ and $0.71 \%$, respectively. The accuracy of the method was calculated after nine injections of known concentration of baicalin, baicalein and wogonin $(50 \mu \mathrm{g} / \mathrm{mL})$ were carried out on three different days and were compared with the measured concentration (baicalin: $50 \pm 4.61$ $\mu \mathrm{g} / \mathrm{mL}$; baicalein: $50 \pm 4.34 \mu \mathrm{g} / \mathrm{mL}$; wogonin: 50 $\pm 0.27 \mu \mathrm{g} / \mathrm{mL}$ ).

This together with the low standard deviation observed supported the accuracy of the analytical method. CVs smaller than $\pm 15 \%$ of the measured value with respect to the known value reflect good accuracy of the method (23). The limit of detection (LOD) (S/N: 3.3) for baicalin, baicalein and wogonin using the developed method were $2.20 \mathrm{ng} / \mathrm{mL}, \quad 3.32 \mathrm{ng} / \mathrm{mL}$ and $4.34 \mathrm{ng} / \mathrm{mL}$, respectively. The limit of quantification (LOQ) (S/N: 10) for baicalin, baicalein and wogonin using the developed method were $6.68 \mathrm{ng} / \mathrm{mL}$, $10.06 \mathrm{ng} / \mathrm{mL}$ and $13.16 \mathrm{ng} / \mathrm{mL}$, respectively.

\section{DISCUSSION}

Analytical techniques for separation and analysis of Scutellaria baicalensis active components have been recently reviewed (24). Reversed-phase C18 columns were most common, and mobile phases usually consisted of an acidic buffer to suppress ionisation of the acidic groups and an organic solvent (commonly methanol or acetonitrile). For example, $0.1 \%$ phosphoric acid/acetonitrile has been used in a 35 min gradient up to $90 \%$ acetonitrile (25). Another investigation originally used a phosphate buffer of $\mathrm{pH} 3.3$ and methanol, but was improved with changing to acetic or formic acid at $\mathrm{pH} 2.5-3.0$ and methanol (26). Among later investigations, $0.1 \%$ formic acid/acetonitrile was used with a gradient from 30 to $85 \%$ acetonitrile in $16 \mathrm{~min}$ (27), or an isocratic mixture of methanol $/ 0.1 \%$ formic acid $(60: 40 \mathrm{v} / \mathrm{v})$ for LC-MS analysis (28). Others used $0.2 \%$ acetic acid/acetonitrile up to $60 \%$ acetonitrile over 55 min and found that retention behaviour of flavonoids on a reversed phase column was remarkably affected by the $\mathrm{pH}$ of the mobile phase (29).

In the present study, the importance of correct acid concentration emerged after initial chromatographic runs with $0.01 \%$ formic acid (measured $\mathrm{pH}$ 2.91)/methanol, with isocratic elution at $40 \%$ aqueous: $60 \%$ organic. While baicalein and wogonin eluted in that order from the column in line with previous reported results, baicalin eluted relatively later in the chromatogram with poor peak shape. In previous studies baicalin eluted early, and our tests varying the acidity of the mobile phase showed that if the formic acid concentration was raised to $0.1 \%$ (measured $\mathrm{pH}$ 2.62) baicalin eluted before the other two with good peak shape.

Table 1. The concentration $(\mu \mathrm{g} / \mathrm{ml})$ of baicalin, baicalein and wogonin present in 7 samples of Scutellaria lateriflora tinctures (SL1-7) and four samples of Scutellaria baicalensis tinctures (SB1-4). All values are mean $\pm \mathrm{SD}(\mathrm{n}=3)$ and are to 3 significant figures. The limits of detection (LOD) are $0.002,0.003$ and $0.004 \mu \mathrm{g} / \mathrm{mL}$ respectively for baicalin, baicalein and wogonin.

\begin{tabular}{lccc} 
& Baicalin & Baicalein & Wogonin \\
\hline SL1 & $<$ LOD & $280 \pm 1.3$ & $<$ LOD \\
SL2 & $180 \pm 0.8$ & $310 \pm 1.5$ & $119 \pm 0.8$ \\
SL3 & $<$ LOD & $<$ LOD & $<$ LOD \\
SL4 & $199 \pm 0.3$ & $347 \pm 1.0$ & $157 \pm 0.5$ \\
SL5 & $268 \pm 0.3$ & $375 \pm 0.8$ & $97.5 \pm 0.4$ \\
SL6 & $<$ LOD & $276 \pm 1.5$ & $<$ LOD \\
SL7 & $12700 \pm 60$ & $629 \pm 11$ & $152 \pm 0.5$ \\
SB1 & $7270 \pm 111$ & $5880 \pm 57$ & $1610 \pm 3$ \\
SB2 & $177 \pm 2.1$ & $4430 \pm 3$ & $112 \pm 2.9$ \\
SB3 & $10600 \pm 190$ & $667 \pm 2.8$ & $262 \pm 4.0$ \\
SB4 & $117 \pm 0.1$ & $517 \pm 1.0$ & $80.0 \pm 0.7$ \\
\hline
\end{tabular}


This acid concentration was therefore adopted for the reported HPLC protocol. Tests were carried out to optimize a gradient elution to achieve sufficient resolution between the selected biomarkers in the tincture products in order to obtain accurate quantitation while maintaining an acceptable gradient time. Few papers using HPLC-UV methods have included data regarding the lower limit of quantification (LLQ) (30-33). Our analytical method shows better sensitivity when compared to these other studies and has a comparable LLQ with LC-MS (29).

Concerning Scutellaria liquid extracts and tinctures, herbal practitioners in the UK are thus faced with a wide range varying widely in the drug-to-solvent ratio (1:1 to 1:5) depending on the manufacturer (2). The concentration of alcohol used typically ranges from $70 \%$ down to $25 \%$. Taken together, this variability in drug-to-extract ratio and the range of alcohol concentrations may partly explain the differential extraction of plant constituents, a common source of variability between commercial preparations. Variability within and between batches can result from different methods of cultivation, extraction, seasonal factors, time of harvesting, storage conditions and natural intraspecific variation in Scutellaria herb. A further compounding factor is the manufacturing process: cold maceration or percolation being two preferred methods (2). The quantitative data on the three biomarkers for $S$. lateriflora tinctures sourced from herbal suppliers used by UK herbal practitioners thus provide interesting results. According to the HPLC chromatograms (Figure 2), the products appear to fall into 3 distinct groups: SL7 is unique with higher concentrations of the biomarkers, particularly baicalin $(12.66 \mathrm{mg} / \mathrm{mL}$ baicalin; 0.63 $\mathrm{mg} / \mathrm{mL}$ baicalein; $0.15 \mathrm{mg} / \mathrm{mL}$ wogonin) compared to SL1-6 (0 - 0.27 mg/mL baicalin; 0 $0.37 \mathrm{mg} / \mathrm{mL}$ baicalein; $0-0.20 \mathrm{mg} / \mathrm{mL}$ wogonin). It is notable that SL7 was the only product extracted in $45 \%$ ethanol compared to $25 \%$ for the other 6 products. Studies in our laboratory have confirmed that $45 \%$ ethanol extracts up to 5 -fold more baicalein and 2-fold more baicalin than $25 \%$ ethanol for the same batch of herbal material. By contrast to SL7, the products SL2, 4 and 5 have similar chromatographic profiles not only in terms of the qualitative and quantitative data for the biomarkers, but also for other unidentified HPLC peaks present in similar proportions. Finally, the products SL1, 3 and 6 are distinguished by the lack of any appreciable biomarker content, but the presence of an unidentified HPLC peak at 11.9 min. The HPLC chromatograms for S. baicalensis tinctures show similar variability in the biomarker content across the four products analysed (Figure 3). Product SB4 appears to be lacking any appreciable biomarker content compared to SB1-SB3, which have similar qualitative profiles. The absence of baicalin is striking, as it is consistently the main biomarker identified in published chromatograms. It is also of note that the same manufacturer produced both SL7 and SB4, yet the SL7 product had higher concentrations of baicalin than competitors, whereas the SB4 product was notably lacking in any biomarker content by comparison with competitors.

Importantly, although GMP regulations cover the manufacturing process they do not necessarily specify the quality or source of the starting plant material. This state of regulation has therefore led to the wide range in quality of Scutellaria products that face herbal practitioners in the UK due to plant material sourced within the UK and imported from countries including the Lebanon and the USA (S. lateriflora) and China (S. baicalensis). Implications of quality, seasonal variation and cost are obviously paramount to manufacturers and may even entail sourcing from different countries on a year-by-year basis, depending on availability of quality material. Interestingly, Han et al showed that $70 \%$ methanol extracts of $S$. baicalensis collected from six different regions of China gave virtually identical chromatograms by electrospray-MS analysis (29). Concerning the \% ethanol used to prepare extracts, these products ranged from drug to solvent ratios of $1: 1$ to $1: 5$ and from $25 \%$ to $70 \%$ ethanol. For these commercial products, no correlation between the concentration of ethanol used and concentration of biomarker was observed and further experiments using the same herbal material would be required to investigate the effects of $\%$ ethanol and drug to solvent ratios on efficiency of biomarker extraction. Clearly, the use of higher \% ethanol, if proven to extract more of the biomarkers, would have cost implications for manufacturers. Further research on the efficacy of S. lateriflora as an anxiolytic and studies on the bioavailability of the biomarkers may be required to convince many manufacturers to use higher \% ethanol extracts. Another important consideration is herbal practitioners' preference for using low alcohol extracts for young, elderly and hepatically-impaired patients. 
The wide variability in biomarker content seen here for Scutellaria species reflects our previous study on the wide variability of biomarker content (rosmarinic acid) for tinctures made from both fresh and dried lemon balm (Melissa officinalis) (20). Two major concerns for manufacturers are the authentication of plant material and the shelf-life of tincture products. Commercial skullcap (S. lateriflora) has in the past been adulterated with Teucrium species, which has been associated with hepatotoxicity at certain doses, perhaps as a result of drug interactions $(1,5)$. Although Gaffner et al reported teucrioside as a marker compound for Teucrium canadense L. and T. chamaedrys L. (34), there is as yet no HPLC-UV method to definitively authenticate $S$. lateriflora and much further research is needed. A further concern is that the shelf life of tincture products is under-researched. Gafner and Bergeron (35) provide an excellent review of the environmental factors affecting chemical stability of tinctures include $\mathrm{pH}$, light and temperature. The presence of air in tincture products can lead to oxidation whilst the presence of metal ions mainly iron, copper and other transition metals, may cause hydrolysis and oxidation reactions with redox active molecules. Amber vials widely used to store tincture products contain significantly higher amounts of iron and titanium and may lead to leaching of metals leading to oxidation over a long storage period. Similarly, the presence of high levels of water has been shown in many studies to cause a more rapid decrease in important biomarker compounds. This could lead to differences in shelf-life between products containing $25 \%$ up to $70 \%$ ethanol. In particular, Gafner and colleagues have reported the degradation of flavone-glucuronides by glucuronidases in fresh $S$. lateriflora extracts, where a combination of heat treatment and addition of antioxidants was required to stabilise extracts. Little systematic research has been done on the shelf-life of tincture products, and this parameter may be a source of variability amongst the products we tested. An important paper by Bilia and colleagues (36) reported a half life of only 3 months for the purported bioactive silymarin complex in alcoholic tincture products of Milkthistle, as measured by LC-DAD and LC-MS. This has serious implications for storage and labeling of tincture products.

Herbal practitioners rely to a large degree on anecdotal evidence in their prescribing of plant remedies due to a paucity of scientific research. However, the professional regulation of herbal practice is under review in the UK, and with the underlying principle of evidence-based practice informing primary healthcare, qualification of herbal practitioners arguably should involve an understanding of the pharmacological and pharmacodynamic basis of herbal medicine. There is therefore a pressing need to establish a scientific evidence base for the practice of herbal medicine to help herbalists to be more discriminatory in their choice of products. Additionally, in regard to quality, with currently no standardised production methods for the manufacture of tinctures used by herbalists there is no guarantee of quality or efficacy. One of the products tested here (SL7) would provide a daily dose of baicalin equivalent to $50 \mathrm{mg}$, compared to other tincture products that contain none. While it is not yet known whether baicalin is solely responsible for a putative anxiolytic activity, nor is the bioavailability in humans reported, this data is clearly important in deciding which products and what dose to use for further clinical research. There is thus an urgent need not only for a review by manufacturers of their production procedures for the herbs formulated as tinctures popularly used by herbal practitioners, but also for educating herbal practitioners to relate quality and efficacy. Clearly, for properly designed clinical trials to provide meaningful data, the method of preparation and quality of tincture products is critical in determining the efficacy of a given treatment. The data presented here provides valuable information as to which Scutellaria tincture products may be suitable for testing in vitro anxiolytic activity and for pilot clinical studies as an anxiolytic treatment.

\section{CONCLUSION}

Wide variability in the biomarker content of herbal preparations undermines the practice of herbal medicine. There is an urgent need for products to be labeled with accurate assessment of the content of agreed biomarkers. Authors, reviewers and editors responsible for reports of pharmacological and clinical activities of herbal products should ensure that the biomarker content of the product used is stated clearly. Where this ideal is not feasible full details of the source and the methods used in its preparation should be included for the benefit of future reviewers and meta-analysis. 


\section{ACKNOWLEDGEMENTS}

The authors wish to thank Biswajit Chowdhury, Meenakshi Ingole and Tania Luraschi for preliminary work and Mrs Julia Freeman for excellent technical support. J. Gao acknowledges a PhD Scholarship from the School of Health and Biosciences, University of East London and is grateful for financial assistance from the Sir Richard Stapley Educational Trust.

\section{REFERENCES}

[1]. Barnes J, Anderson L and Phillipson JD. (2002). Herbal Medicines: A guide for healthcare professionals. $2^{\text {nd }}$ Edition. Pharmaceutical Press, London.

[2]. Bone K. (2003). A clinical guide to blending liquid herbs. Churchill Livingstone, Edinburgh.

[3]. British herbal pharmacopoeia (1996). $4^{\text {th }}$ Edition, British Herbal Medicine Association.

[4]. Awad R, Arnason JT, Trudeau V, Bergeron C, Budzinski J.W, Foster B. C and Merali Z. (2003). Phytochemical and biological analysis of Skullcap (Scutellaria lateriflora L): A medicinal plant with anxiolytic properties. Phytomedicine 10(8): 640-649.

[5]. Wolfson PE and Hoffman DL. (2003). An investigation into the efficacy of Scutellaria lateriflora in healthy volunteers. Alternative therapies in health and medicine, 9(2): 74-78.

[6]. Konoshima T, Kokumai M, Kozuka M, Iinuma M, Mizuno M, Tanaka T, Tokuda H, Nishino H and Iwashima A. (1992). Studies on inhibitors of skin tumour promotion. XI. inhibitory effects of flavonoids from Scutellaria baicalensis on Epstein-Barr virus activation and their anti-tumour-promoting activities. Chemical \& Pharmaceutical Bulletin 40(2): 531-533.

[7]. Chan F.L, Choi HL, Chen Z.Y, Chan P.S and Huang Y. (2000). Induction of apoptosis in prostate cancer cell lines by a flavonoid, baicalin. Cancer Letters 160(2): 219-228.

[8]. Ikemoto S, Sugimura K, Yoshida N, Yasumoto $\mathrm{R}$, Wada S, Yamamoto $\mathrm{K}$ and Kishimoto $\mathrm{T}$ (2000). Antitumor effects of Scutellaria radix and its components baicalein, baicalin, and wogonin on bladder cancer cell lines. Urology 55(6): 951-955.

[9]. Koda A, Nagai H and Wada H. (1970). Pharmacological actions of baicalin and baicalein (Report 1) On the active anaphylaxis. Folia Pharmacologica Japonica 66(2): 194-213

[10]. Koda A, Nagai H and Wada H. (1970). Pharmacological actions of baicalin and baicalein (Report 2) On the passive anaphylaxis Folia Pharmacologica Japonica 66(2):
237-247.

[11]. Koda A, Nagai H and Wada H. (1970). Pharmacological actions of baicalin and baicalein (Report 3) Action on experimental asthma. Folia Pharmacologica Japonica 66(5): 471-486.

[12]. Lin C.C and Shieh D.E. (1996). The anti-inflammatory activity of Scutellaria rivularis extracts and its active components, baicalin, baicalein and wogonin. The American Journal of Chinese Medicine 24(1): 31-36.

[13]. Li B.Q, Fu T, Gong W.H, Dunlop N, Kung H, Yan Y.D, Kang J and Wang J.M (2000). The flavonoid baicalin exhibits anti-inflammatory activity by binding to chemokines. Immunopharmacology 49(3): 295-306.

[14]. Shieh DE Liu LT and Lin CC. (2000). Antioxidant and free radical scavenging effects of baicalein, baicalin and wogonin. Anticancer Research 20(5A): 2861-2865.

[15]. Gao ZH, Huang KX and Xu HB (2001). Protective effects flavonoids in the roots of Scutellaria baicalensis Georgi against hydrogen peroxide-induced oxidative stress in HS-SY5Y cells. Pharmacological Research 43(2): 173-178.

[16]. Bochorakova H, Paulova H, Slanina J, Musil P and Taborska E (2003). Main flavonoids in the root of Scutellaria baicalensis cultivated in Europe and their comparative antiradical properties. Phytotherapy Research 17(6): 640-644.

[17]. Wu J.A, Attele AS, Zhang L and Yuan C.S (2001). Anti-HIV activity of medicinal herbs: usage and potential development. The American Journal of Chinese Medicine 29(1): 69-81.

[18]. Himeji M, Ohtsuki T, Fukazawa H, Miho T, Yazaki S, Ui S, Nishio K, Yamamoto, Tasaka $\mathrm{K}$ and Mimura A. (2006). Difference of growth-inhibitory effect of Scutellaria baicalensis-producing flavonoid wogonin among human cancer cells and normal diploid cell. Cancer Letters 245(1-2): 269-274.

[19]. Huang R.L, Chen C.C, Huang H.L, Chang C.G, Chen C.F, Chang C and Hsieh M.T (2000). Anti-hepatitis B virus effects of wogonin isolated from Scutellaria baicalensis. Planta Medica 66(8):694-698.

[20]. Sanchez-Medina A, Etheridge CJ, Hawkes GE, Hylands PJ, Pendry BA, Hughes MJ and Corcoran O. (2007). Comparison of rosmarinic acid content in commercial tinctures produced from fresh and dried lemon balm (Melissa officinalis). J. Pharm. Pharmaceut. Sci. 10(4): 455-463.

[21]. Webb GP. (2006). Dietary supplements and functional foods. Blackwell Publishing, Oxford.

[22]. Webb GP. (2007). Nutritional supplements and 
conventional medicine; what should the physician know? Proceedings of the Nutrition Society, 66, 471-478.

[23]. Rosing H, Man WY, Doyle E, Bult A and Beijnen JH. (2000) Bioanalytical liquid chromatographic method validation. A review of current practices and procedures. Journal of liquid chromatography and related technologies. 23: 329-354.

[24]. Li H.B, Jiang Y and Chen F. (2004). Separation methods used for Scutellaria baicalensis active components. Journal of Chromatography B. 812 (1-2): 277-290.

[25]. Lin MC, Tsai MJ, and Wen KC. (1999). Supercritical fluid extraction of flavonoids from Scutellaria radix, Journal of Chromatography A 830, 387-395.

[26]. Cai Y., Luo Q, Sun M and Corke H (2004). Antioxidant activity and phenolic compounds of 112 traditional Chinese medicinal plants associated with anticancer, Life Sciences 74, 2157-2184

[27]. Horvath CR, Martos PA and Saxena PK (2005). Identification and quantification of eight flavones in root and shoot tissues of the medicinal plant Huang-qin (Scutellaria baicalensis Georgi) using high-performance liquid chromatography with diode array and mass spectrometric detection, Journal of Chromatography A 1062, 199-207.

[28]. Kim YH, Jeong DW, Paek IB, Ji HY, Kim YC, Sohn DH and Lee HS (2006) Liquid chromatography with tandem mass spectrometry for the simultaneous determination of baicalein, baicalin, oroxylin A and wogonin in rat plasma, Journal of Chromatography B 844, 261-267.

[29]. Han J, Ye M, Xu M, Sun J, Wang B and Guo D (2007). Characterization of flavonoids in the traditional Chinese herbal medicine-Huangqin by liquid chromatography coupled with electrospray ionization mass spectrometry, Journal of Chromatography B 848, 355-372.

[30]. Lai M, Hsiu S, Chen C, Hou Y and Chao PL. (2003). Urinary Pharmacokinetics of baicalein, wogonin and their glycosides after oral administration of Scutellaria Radix in humans. Biol. Pharm. Bull 26(1): 79-83

[31]. Zhang L, Lin G and Zuo Z. (2004). High-performance liquid chromatographic method for simultaneous determination of baicalein and baicalein 7-glucuronide in rat plasma. Journal of Pharmaceutical and Biomedical Analysis 36: 637-641.

[32]. Lu T, Song J, Huang F, Deng Y, Xie L, Wang G and Liu X. (2006). Comparative pharmacokinetics of baicalin after oral administration of pure baicalin, Radix scutellaria extract and Huang-Lian-jie-Du-Tang to rats. Journal of Ethnopharmacology 110:412-418.

[33]. Yu K, Gong YF, Lin ZY and Cheng YY. (2007). Quantitative analysis and chromatographic fingerprinting for the quality evaluation of Scutellaria baicalensis Georgi using capillary electrophoresis. Journal of Pharmaceutical and Biomedical Analysis 43: 540-548.

[34]. Gafner S, Bergeron C, Batcha LL, Angerhofer CK, Sudberg S, Sudberg EN, Guinaudreau H and Gauthier R. (2003). Analysis of Scutellaria lateriflora and its adulterants Teucrium canadense and Teucrium chamaedrys by LC-UV/MS, TLC and digital photomicroscopy. Journal of AOAC International, 86(3): 453-460.

[35]. Gafner S and Bergeron C. (2005) The challenges of chemical stability testing of herbal extracts in finished products using state-of-the-art analytical methodologies. Current Pharmaceutical Analysis, 1, 203-215.

[36]. Bilia AR, Bergonzi MC, Gallori S, Mazzi G and Vincieri FF. (2002) Stability of the constituents of calendula, milk thistle and passionflower tinctures by LC-DAD and LC-MS. Journal of Pharmaceutical and Biomedical Analysis. 30: 613-624. 